\title{
A Brief Online Mindfulness-Based Group Intervention for Psychological Distress Among Chinese Residents During COVID-19: a Pilot Randomized Controlled Trial
}

\author{
Hui Zhang ${ }^{1}$ (I) $\cdot$ Anao Zhang $^{2} \cdot{\text { Chengbin } \mathrm{Liu}^{1} \cdot \text { Jian Xiao }}^{3} \cdot$ Kaipeng Wang $^{4}$ \\ Accepted: 3 March 2021 / Published online: 18 March 2021 \\ (C) The Author(s), under exclusive licence to Springer Science+Business Media, LLC, part of Springer Nature 2021
}

\begin{abstract}
Objectives The coronavirus (COVID-19) global pandemic has increased psychological distress among the general population. The objective of this study is to evaluate a mindfulness-based intervention for psychological distress among Chinese residents during COVID-19.

Methods This study used a switching replications design to test the feasibility and efficacy of a brief online mindfulness-based intervention for Chinese residents' psychological distress. Fifty-one residents in the Hubei province were randomly allocated to two groups (experimental group and waitlist control group) with three waves of measurement at time 1, time 2, and time 3 for changes in mindfulness and psychological distress.

Results In addition to significant within-group improvements over time for both groups, OLS linear regression with full information likelihood estimation revealed statistically significant between-group treatment effects across outcome domains, including mindfulness awareness, $b=2.84, p<0.001, g=6.92$, psychological distress, $b=-21.33, p<0.001, g=6.62$, somatic symptoms, $b=-6.22, p<0.001, g=4.42$, depressive symptoms, $b=-7.16, p<0.001, g=5.07$, and anxiety symptoms, $b=$ $-8.09, p<0.001, g=6.84$.

Conclusions Results suggest that a brief online mindfulness-based intervention can be a feasible and promising intervention for improving mindfulness and decreasing psychological distress among Chinese residents staying at home during the COVID-19 outbreak. The study used a small convenience sample which led to a concern of external generalizability and with limited evaluation of long-term change.
\end{abstract}

Keywords COVID-19 · Mindfulness-based intervention · Online intervention $\cdot$ Psychological distress

The global outbreak of the coronavirus (COVID-19) pandemic continues to rapidly unfold every day, with shelter-in-place orders and strict social distancing policies remaining across the world. In China, a strict quarantine order has been adopted in the Hubei province since 23 January 2020 and was only recently

Hui Zhang

zhanghui_0927@hust.edu.cn

1 School of Sociology, Huazhong University of Science and Technology, 1037, Luoyu Road, Hongshan District, Wuhan, China

2 School of Social Work, University of Michigan, Ann Arbor, MI, USA

3 Sinopharm Dongfeng General Hospital, Shiyan, China

4 Graduate School of Social Work, University of Denver, Denver, CO, USA lifted. In addition to the concerning physical health consequences of potentially contracting COVID-19, the general population, especially during strict quarantine and social distancing mandates, also suffers from major psychological distress, like depression, anxiety, and possible somatic symptoms (Li et al., 2020). Psychological distress is defined as "the unique discomforting, emotional state [like depression or anxiety] experienced by an individual in response to a specific stressor or demand ... to the person" (Ridner, 2004, p. 539). Factors contributing to high levels of psychological distress are mainly twofold. First, due to the novelty of this virus and the absence of an effective vaccine and medical treatment, health anxieties (e.g., fear and chronic worry) are often high among the general population.

Consequently, and concurrently, somatic symptoms (e.g., chest pains and shortness of breath) and obsessive-compulsive behaviors (e.g., repeated temperature checks and over-sterilizations) become far more frequent and prevalent than the pre- 
pandemic period (Bao et al., 2020; Shigemura et al., 2020). Second, due to the broad implementation of the stay-at-home order, most of the Chinese peoples' lives were abruptly interrupted by mandated social isolation, imposed indoor restrictions, and forced them to miss out on significant life events, such as weddings and baby showers (Li et al., 2020). As a result, individuals are at high risk of developing depressive and/or anxiety symptoms as well as high levels of psychological distress.

Empirically, a recent study reported by the Chinese Social Survey ( $N=12,448$ residents in Hubei, China) found that $87 \%$ and $75 \%$ of the participants reported fear and anxiety, respectively, due to COVID-19 and self-isolation (Gao, 2020). Another study of 1210 participants from 194 cities in China revealed that $53.8 \%$ of participants reported the psychological impact of the outbreak of COVID-19 as substantial, with $16.5 \%$ reporting moderate to severe depressive symptoms, $28.8 \%$ reporting moderate to severe anxiety symptoms, and $8.1 \%$ reporting moderate to severe stress levels (Wang et al., 2020a, b).

If unaddressed, severe psychological distress may lead to adverse physical and mental health outcomes for residents, especially during the stay-at-home period. First, under a state of high psychological distress, individuals may have lower fruit and vegetable intake, greater waist-to-height ratio, compromised food security (Davison et al., 2020; Wang et al., 2020a, b; Wang \& Bishop, 2019), and have a higher probability of suffering from cognitive impairment and physical frailty (Jing et al., 2020), which may damage individuals' immune system to increase the risk of contracting a disease, such as the COVID-19. Furthermore, previous studies suggest that psychological distress, especially persistent distress, may be a risk factor for incident dementia (Nakamura et al., 2019) and functional disability (Tanji et al., 2017), further highlighting the debilitating consequences of unaddressed psychological distress.

Second, psychological distress may reduce individuals' ability to cope, further exacerbating their already compromised mental health and overall quality of life (Coffey \& Hartman, 2008). For example, previous studies indicate that psychological distress is related to increased risk of severe mental disorders, such as major depressive disorder (Chisholm et al., 2016; Nishi et al., 2018), further highlighting the significance of timely intervention for psychological distress to support the general population (Li et al., 2020). In acknowledging the salient mental health concern during a global pandemic, the National Health Commission of China (NHC) had, early on, put forth a nationwide guideline that a psychological crisis intervention be taken into consideration for the general deployment of disease prevention during the COVID-19 pandemic ( $\mathrm{Li}$ et al., 2020). One promising intervention is the mindfulness-based treatment for psychological distress.
Theoretical and clinical literature addressing psychological distress has focused on individuals' attention to and repetitive negative thinking about distressful events (Figueroa et al., 2019; Mazzer et al., 2019). As a result, a critical component that facilitates the therapeutic progress of psychological distress is to modify individuals' cognitive evaluations of the distressful event and to discontinue their subsequent negative thoughts afterward. One such component that has received strong theoretical and empirical support for coping with psychological distress is mindfulness (Ma et al., 2018; Zhang et al., 2020a, b).

Mindfulness helps individuals focus on the present in a non-judgmental way, break the constant connectivity to one's malfunctioning cognitive patterns, and disengage oneself from existing perceptions, emotions, and distressful behaviors (Baer, 2010; Garland et al., 2015; Langdon et al., 2011; Shapiro et al., 2006). For example, the overwhelming news reporting of COVID-19 may be a constant reminder for stayat-home residents that is likely to trigger psychological distress. Through the use of mindfulness, residents may appraise this news reporting differently and, consequently, distance themselves from persisting health worries and anxieties. Therefore, it is reasonable to expect that the use of and engagement in mindfulness is likely to alleviate psychological distress among Chinese residents who are staying at home.

Given the critical role of mindfulness in redirecting attention from anxiety-provoking COVID-19 to an alternative and neutral appraisal of the global pandemic, the intervention aims to enhance awareness of and pay attention to the present and break the continuous negative rumination about COVID-19 to relieve psychological distress. The practice of paying attention to the present, through a mindfulness-based intervention, plays an essential role in reducing depressive symptom severity (Lönnberg et al., 2020; Shapiro et al., 1998), anxious mood (Kabat-Zinn, 1982; Watanabe et al., 2019), rumination (Chiesa et al., 2014; Deyo et al., 2009), and psychological distress (Perez-Blasco et al., 2013).

Due to the strict social distancing policies, in-person mental health interventions are not feasible. With the rapid development of technology in mental health, online mental health interventions have amassed a large body of literature supporting their effectiveness for mental health outcomes (Zhang et al., 2019). Clinical trials and meta-analyses have consistently reported favorable findings on the treatment effect of online mindfulness-based interventions to improve clients' mental health and stress management (Spijkerman et al., 2016; Zhang et al., 2020a, b).

In this current study, we tested the following hypotheses: (1) Participants in the experimental group receiving the online mindfulness-based intervention (between $\mathrm{T} 1$ and $\mathrm{T} 2$ ) will report a higher level of mindfulness, lower level of distress, somatization, depression, and anxiety than participants in the waitlist control group (waitlist control) at T2; (2) Participants 
in the waitlist control group receiving the online mindfulnessbased intervention (between T2 and T3) will report no differences than those in the experimental group (waitlist control between T2 and T3) for mindfulness, distress, somatization, depression, and anxiety; and (3) Participants in both groups will report significant improvements between $\mathrm{T} 1$ and $\mathrm{T} 3$ for mindfulness, distress, somatization, depression, and anxiety.

\section{Methods}

\section{Participants}

Participants were residents in Hubei province, China, and stayed at home during the novel coronavirus outbreak from 25th January to 14th March per the government's shelter-inplace order. Participants were recruited through convenient snowball sampling. Initial contacts were made through an advertising webpage with the purpose, procedures, and inclusion/exclusion criteria of the study. The recruiting webpage was distributed through the study team's social network using WeChat. Those interested in the study were asked to contact the study team to answer the online questionnaires as an initial screening. Eligible and consented participants (defined below) reported their demographic information, psychological distress, and mindfulness at time 1 (baseline).

\section{Inclusion and Exclusion Criteria}

To be eligible for participation, a prospective participant needed to: (1) be aged 21 years or older; (2) meet the genderspecific baseline threshold score ( $>10$ for males and $>13$ for females) of the Chinese version Brief Symptom Inventory-18 (BSI-18) (Wang et al., 2013); and (3) have a smartphone, laptop, or desktop computer to receive the online intervention. We excluded any participants who had severe physical disease or cognitive impairment or were unwilling to provide consent. We also would exclude any participants who were receiving an on-going technology-based mental health intervention.

After excluding four participants who did not meet the criteria, 57 participants were recruited who provided voluntary consent and agreed to complete all study components. Participants were randomly assigned to two groups, the experimental group $(n=29)$ and the waitlist control group $(n=28)$. Four participants in the experimental group and two participants in the waitlist control group dropped out for reasons indicated in the CONSORT chart (Fig. 1).

Participants had a mean age of $50.12(\mathrm{SD}=6.79)$, approximately half ( $n=24,47.06 \%)$ were female, and most of them had a middle school degree $(n=38,74.51 \%)$. On average, based on an algorithm of the BSI-18 scoring manual (Recklitis et al., 2017), participants (from both groups) reported moderately severe psychological distress $($ mean $=31.61, \mathrm{SD}=3.77$ ) and low mindfulness-awareness $($ mean $=2.11, \mathrm{SD}=0.25$ ). More specifically, participants at baseline (T1) reported a moderately high level of anxiety (mean $=12.33, \mathrm{SD}=2.28$ ) followed by moderate depressive symptoms (mean $=9.82$, SD $=1.14)$ and moderate somatic symptoms (mean $=9.45, \mathrm{SD}=$ 1.67). No demographic or clinical characteristic information was statistically different between the experimental group and waitlist control group, which supported the validity of randomization. Throughout the study, no participants reported concerns about self-harm or harming others. Table 1 presents detailed demographic and clinical characteristic information at baseline (Time 1).

\section{Procedures}

This study used an experimental switching replication design to (1) allow all participants to have the opportunity to receive intervention while simultaneously (2) ensuring a methodologically robust design to evaluate the intervention's treatment effect. The switching replication design includes two groups with three wave measurements at time 1, time 2, and time 3 (Fig. 1). Using a computer random number generator overseen by an independent statistician, participants were randomly assigned to one of two groups (labeled as experimental group and waitlist control group). Participants in the experimental group received the intervention between time 1 and time 2, during which participants in the waitlist control group served as waitlist control. The statistician and the lead data analyst were blind to participants' assignment. Given the nature of the intervention, we were unable to blind study participants, the psychologist, and the outcome assessor (research staff).

Similarly, participants in the waitlist control group received the intervention between time 2 and time 3, during which experimental group participants served as waitlist control. For each time point, i.e., time 1 to time 3 , an individual participant scheduled an appointment with a trained research staff for a 30-min virtual session completing all assessment measures (described below) and a question asking about their risk of self-harming and/or harming others. We were successful in scheduling assessment sessions with all participants on the day of their scheduled timeline.

\section{Intervention Group}

The intervention tested in this study was based on the Mindfulness-Based Stress Reduction program (MBSR) developed by Kabat-Zinn (1982). The original MBSR program includes weekly 2.5 -h small group classes for 8 weeks plus a half-day meditation retreat. The original version of MBSR has been widely supported by empirical literature (de Vibe et al., 2017; Sharma \& Rush, 2014); however, there have been discussions on the program's high duration and intensity, 


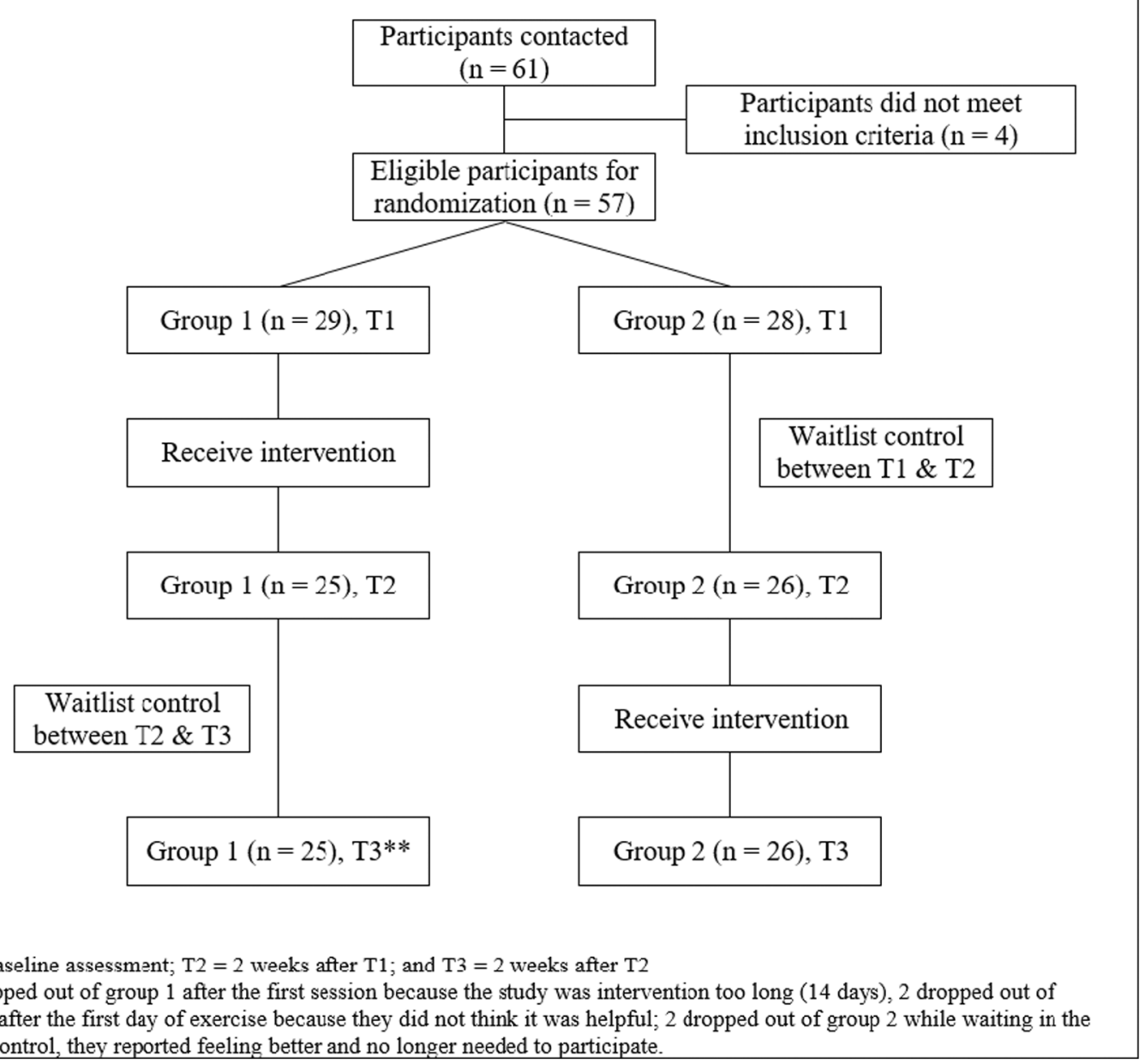

Fig. 1 CONSORT flow diagram

Table 1 Group comparison of demographics and clinical characteristics at time 1

\begin{tabular}{|c|c|c|c|c|c|c|c|}
\hline \multirow[t]{2}{*}{ Variable } & \multicolumn{2}{|l|}{ Total } & \multicolumn{2}{|c|}{ Group 1} & \multicolumn{2}{|c|}{ Group 2} & \multirow{2}{*}{$\begin{array}{l}\text { Between group difference }(t \text { test ol } \\
\left.\chi^{2}\right)^{*}\end{array}$} \\
\hline & M & $\mathrm{SD}$ & M & SD & M & $\mathrm{SD}$ & \\
\hline Age & 50.12 & 6.61 & 50.14 & 6.51 & 50.11 & 6.83 & 0.02 \\
\hline \multicolumn{8}{|l|}{ Gender** } \\
\hline Male & 29 & & 13 & & 16 & & \multirow[t]{2}{*}{0.86} \\
\hline Female & 28 & & 16 & & 12 & & \\
\hline \multicolumn{8}{|l|}{ Educational level } \\
\hline Middle school & 42 & & 21 & & 21 & & \multirow[t]{3}{*}{0.07} \\
\hline High school & 11 & & 6 & & 5 & & \\
\hline$\geq$ College degree & 4 & & 2 & & 2 & & \\
\hline $\begin{array}{l}\text { Psychological } \\
\text { distress }\end{array}$ & 31.54 & 3.64 & 31.28 & 4.01 & 31.82 & 3.27 & -0.56 \\
\hline Somatization & 9.47 & 1.59 & 9.38 & 1.54 & 9.57 & 1.67 & -0.45 \\
\hline Depression & 9.84 & 1.11 & 9.83 & 1.07 & 9.86 & 1.18 & -0.10 \\
\hline Anxiety & 12.23 & 2.20 & 12.07 & 2.48 & 12.39 & 1.91 & -0.55 \\
\hline Mindfulness & 2.09 & 0.25 & 2.06 & 0.28 & 2.12 & 0.22 & -0.89 \\
\hline
\end{tabular}

$* \chi^{2}$ analysis was used to test the difference of gender and educational level; $t$ test was used for others

**For categorical variables, i.e., gender and education level, frequency was provided 
which may limit its utility among the general population (Kiburz et al., 2017). Therefore, we intentionally reduced the intensity and duration of the original MBSR in this study, hoping that it will have better utility among our participants.

In the current study, we evaluated a brief online mindfulness-based group intervention, including a 2-h training/psycho-education session on mindfulness, followed by a 13-day group-supported mindfulness practice. In session 1 , participants joined a virtue group through WeChat-a multi-function online platform (similar to WhatsApp in the USA). In addition to psycho-educating participants about mindfulness practices during COVID-19 and concerning psychological distress, the group leader, a PhD-level psychologist, also facilitated ice-breaking activities, rapport-building, and set norms about the online/virtue group. Five key mindfulness-based activities were taught during session one: (1) intentional breathing, (2) dismissing thoughts and bring the mind back to the present, (3) attending to the sensations in one's body, (4) noticing breath traveling to body parts, and (5) being mindful of one's being while doing things, e.g., being mindful and processing details when one gets up and drinks some water (Kiburz et al., 2017).

Upon completing session one, participants were instructed to engage in $1.5 \mathrm{~h}$ of mindfulness practices every day, i.e., $30 \mathrm{~min}$ per practice and three times per day, for the next 13 days. Participants received a daily reminder in the morning about practicing mindfulness throughout the day and were asked to complete a self-monitoring sheet reporting their engagement in mindfulness. Participants had access to the virtue group during the 13 days and shared their experiences about practicing mindfulness to cope with psychological distress. Participants were allowed to share their daily life experiences during the shelter-in-place mandate during the study. The group facilitator would guide these conversions to use mindfulness practice as a helpful coping strategy. The PhD-level psychologist was also available remotely throughout the study to address any immediate concerns or clinical deterioration.

\section{Waitlist Control Group}

For participants in the waitlist control group, they were informed to wait for 14 days before they start the intervention. During the 14-day period, if there was a need or an emergency for the participants in the waitlist control, they could contact the research team for crisis intervention and/or obtain resources for mental health services.

\section{Measures}

\section{Psychological Distress}

We measured individual psychological distress using the Chinese version of the BSI-18 (Wang et al., 2013; Zhang et al., 2018), including three subscales (somatization, depression, and anxiety). The scale contains 18 questions, and some sample questions asked participants about their shortness of breath or feeling worthlessness or feeling tense. Items were rated on a 5-point Likert scale from zero (not at all) to 4 (extremely). The total score, in theory, ranges from zero to 72 , with a higher score suggesting greater severity of psychological distress. Alphas across the three time periods ranged from 0.79 to 0.93 .

\section{Mindfulness Scale}

Mindfulness was measured using the Chinese version of the Mindful Attention Awareness Scale (Brown \& Ryan, 2003; Deng et al., 2012). The scale contains 15 questions. One sample item was "I could be experiencing some emotion and not be conscious of it until sometime later." Items were rated on a 6-point Likert scale that ranged from one (almost never) to six (almost always). All items were reverse coded so that higher scores reflected a higher mindfulness trait. The final score, in theory, ranges from zero to 105 , and we averaged the final score across items resulting in a final theoretical scoring range of zero to 7. Alphas across the three time periods ranged from 0.73 to 0.96 .

\section{Data Analyses}

A minimal sample size of 84 participants was needed to achieve an $80 \%$ power for a moderate treatment effect $(g=$ 0.4 ) using fixed-effect analysis of covariance (ANCOVA) for two groups at a critical alpha level of 0.05 . Although our final sample of participants completing the intervention $(n=51)$ is low, based on power analysis, we consider the sample size acceptable given that this study is a pilot feasibility trial (Browne, 1995). Treatment effects were estimated using ordinary least square (OLS) linear regression, with "time" being the focal predictor for within-group treatment effect and "group assignment" being the focal predictor for betweengroup treatment effect. We also calculated within-group and between-group small sample size corrected Hedges' $g$ to obtain treatment effect size. We conducted an intent-to-treat analysis using full information maximum likelihood (FIML) estimation to address missing values, resulting in a final analytical sample of 57 participants.

\section{Results}

\section{The Practice of Mindfulness}

In addition to the mindfulness-awareness scale, participants were asked to monitor and report their daily mindfulness activities. During the study, participants in the experimental 
group reported, on average, $18.73 \mathrm{~h}(\mathrm{SD}=4.83)$ of practicing mindfulness for 13 days ( 1.44 hours per day), and participants in the waitlist control group reported, on average, $19.01 \mathrm{~h}$ (SD $=4.64)$ of practicing mindfulness for 13 days $(1.46 \mathrm{~h} /$ day $)$. The difference was not statistically significant.

\section{Effects of the Brief, Online-Based Mindfulness Group Intervention}

The treatment effect was analyzed using between-group comparisons (Table 2). The between-group treatment effect at $\mathrm{T} 2$ revealed a statistically significant treatment effect of the brief, online-based mindfulness group intervention for psychological distress compared to the waitlist control. Results of the analysis show that participants in the treatment group reported statistically significant better outcomes in mindfulness-awareness, $b=2.84, p<0.001, g=6.92$, psychological distress, $b=$ $-21.33, p<0.001, g=6.62$, somatic symptoms, $b=-6.22, p<$ $0.001, g=4.42$, depressive symptoms, $b=-7.16, p<0.001, g$ $=5.07$, and anxiety symptoms, $b=-8.09, p<0.001, g=6.84$. Finally, upon waitlist control group participants' completion of their 14-day intervention, the between-group difference was no longer statistically significant. Figure 2 presents a visual representation of participants' progress throughout the study.

Table 3 presents the within-group treatment difference and effect size. Participants in both groups reported statistically significant improvement before and after receiving the intervention. Upon completing the 14-day intervention (between Time 1 and Time 2), the experimental group participants reported significant improvement in their mindfulness awareness, $b=2.86, p<0.001$, total score in psychological distress, $b=-20.80, p<0.001$, somatization, $b=-6.56, p<0.001$, depressive symptoms, $b=-6.92, p<0.001$, and anxiety symptoms, $b=7.32, p<0.001$. The within-group treatment effect remained after the participants finished the 14-day training for at least another 14-days (T3), evidenced by statistically significant improvement between $\mathrm{T} 1$ and $\mathrm{T} 3$ (results presented in Table 2). Within-group small sample size corrected Hedges' $g$ (between T1 and T3) also revealed a statistically significant large treatment effect size across all domains of outcomes, including mindfulness awareness, $g=$ 7.41, $p<0.001$, psychological distress, $g=6.02, p<0.001$, somatization, $g=5.05, p<0.001$, depressive symptoms, $g=$ 5.50, $p<0.001$, and anxiety symptoms, $g=4.07, p<0.001$.

Upon completing the 14-day intervention (between time 2 and time 3), waitlist control group participants reported significant improvement in their mindfulness awareness, $b=$ 2.86, $p<0.001$, total score in psychological distress, $b=$ $-22.96, p<0.001$, somatization, $b=-6.77, p<0.001$, depressive symptoms, $b=-7.19, p<0.001$, and anxiety symptoms, $b=-9.00, p<0.001$. Within-group small sample size corrected Hedges' $g$ also revealed a statistically significant large treatment effect size across all domains of outcomes, including mindfulness awareness, $g=6.36, p<0.001$, psychological distress, $g=7.94, p<0.001$, somatization, $g=$ 5.28, $p<0.001$, depressive symptoms, $g=6.26, p<0.001$, and anxiety symptoms, $g=5.36, p<0.001$.

\section{Discussion}

During the continuing COVID-19 outbreak, residents worldwide, including Chinese residents, are required to stay at home to prevent further transmission of the coronavirus. When faced with this highly contagious virus and the "new norm" during and after COVID-19, Chinese residents, like many of their international peers, face significant psychological distress challenges. Given the significant outcomes of individuals' psychological distress, it is critical to develop and evaluate psychological distress interventions for stay-at-home residents.

In general, this study's findings show that the intervention was effective at increasing mindfulness awareness and simultaneously reducing psychological distress for study participants. For participants in both groups, we observed
Table 2 Between-group treatment effect at $\mathrm{T} 2$ and $\mathrm{T} 3 *$

\begin{tabular}{|c|c|c|c|c|c|c|}
\hline & \multicolumn{3}{|c|}{ Group 1 versus group 2 at $\mathrm{T} 2$} & \multicolumn{3}{|c|}{ Group 1 versus group 2 at T3 } \\
\hline & Adjusted $b$ & SE & Hedges' $g$ & Adjusted $b$ & $\mathrm{SE}$ & Hedges' $g$ \\
\hline Mindfulness & $2.84 * *$ & 0.08 & $6.92 * *$ & -0.07 & 0.08 & 0.10 \\
\hline Distress & $-21.33 * *$ & 0.41 & $6.62 * *$ & 0.58 & 0.38 & 0.23 \\
\hline Somatization & $-6.22 * *$ & 0.23 & $4.42 * *$ & 0.35 & 0.06 & 0.11 \\
\hline Depression & $-7.16^{* *}$ & 0.30 & $5.07^{* *}$ & 0.56 & 0.11 & 0.09 \\
\hline Anxiety & $-8.09 * *$ & 0.39 & $6.84 * *$ & 0.39 & 0.30 & 0.35 \\
\hline
\end{tabular}

*Between-group treatment effect was evaluated controlling for T1 score, age, educational background, and gender. Full information maximum likelihood estimation was used to run the model to address missing value $* * p<0.001$ 
Fig. 2 Line plots about groups 1 and 2
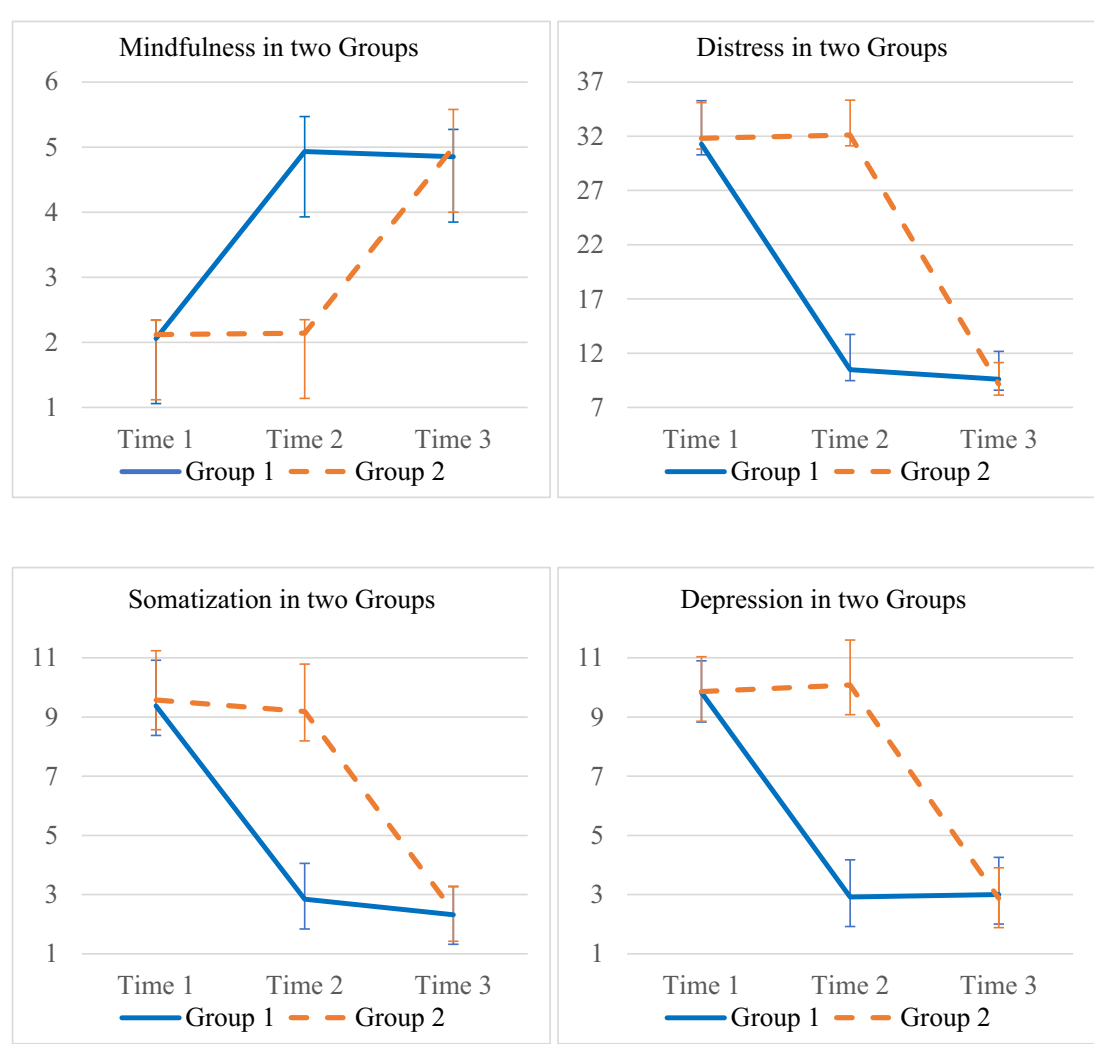

an improvement in mindfulness and a decrease in psychological distress, including all subscales of depression, anxiety, and somatization, between pre- and post-intervention. It is noticeable that improvement in outcomes was clinically meaningful, evidenced by large within-group treatment effect size estimates. Such findings are encouraging as they would suggest real clinical improvements among participants. It is likely that we overestimated the real treatment effect given that the magnitude of the reported withingroup effect size estimates was really large. It was primarily due to the small standard deviation of outcome scores among study participants. As a result, these effect size estimates should be cautiously interpreted for publication bias that is likely to occur in a small sample size pilot study.
Findings in this study are broadly consistent with an existing meta-analysis of online mindfulness-based interventions, which has reported moderate to large treatment effect size for stress management $(g=0.4)$ and mental health $(g=$ 0.5) (Spijkerman et al., 2016; Zhang et al., 2020a, b). Reported treatment effect sizes of online mindfulness-based interventions for stress and mental health outcomes appear to be comparable to other forms of online-based interventions, such as online cognitive behavioral therapy for major depression $(g=0.56)$ and anxiety disorders $(g=0.53)$ (Ahern et al., 2018; Carpenter et al., 2018).

As we conceptualized in our study, the benefits associated with brief mindfulness-based interventions are considered to be a result of increased attention to the present moment, decreased automaticity of thinking, and breaking the link 
Table 3 Within-group treatment effect

\begin{tabular}{|c|c|c|c|c|c|c|c|c|}
\hline & \multicolumn{4}{|l|}{ Group 1} & \multicolumn{4}{|l|}{ Group 2} \\
\hline & Time $1 \mathrm{M} / \mathrm{SD}$ & Time $2 \mathrm{M} / \mathrm{SD}$ & $\mathrm{T} 1$ to $\mathrm{T} 2$ adjusted $b^{*}$ & Hedges' $g$ & Time $1 \mathrm{M} / \mathrm{SD}$ & Time $2 \mathrm{M} / \mathrm{SD}$ & $\mathrm{T} 1$ to $\mathrm{T} 2$ adjusted $b^{*}$ & Hedges' $g$ \\
\hline Mindfulness & $2.06 / 0.28$ & $4.93 / 0.54$ & $2.86 * * *$ & - & $2.12 / 0.22$ & $2.14 / 0.14$ & 0.02 & - \\
\hline Distress & $31.28 / 4.01$ & $10.48 / 3.25$ & $-20.80 * * *$ & - & $31.82 / 3.27$ & $32.12 / 3.20$ & 0.28 & - \\
\hline Somatization & $9.38 / 1.54$ & $2.84 / 1.21$ & $-6.56^{* * *}$ & - & $9.57 / 1.67$ & $9.19 / 1.60$ & -0.38 & - \\
\hline Depression & $9.83 / 1.07$ & $2.92 / 1.26$ & $-6.92 * * *$ & - & $9.86 / 1.18$ & $10.08 / 1.52$ & 0.20 & - \\
\hline \multirow[t]{2}{*}{ Anxiety } & $12.07 / 2.48$ & $4.72 / 1.54$ & $-7.32 * * *$ & - & $12.39 / 1.91$ & $12.85 / 2.05$ & 0.46 & - \\
\hline & Time $2 \mathrm{M} / \mathrm{SD}$ & Time $3 \mathrm{M} / \mathrm{SD}$ & $\mathrm{T} 2$ to $\mathrm{T} 3$ adjusted $b^{*}$ & Hedges' $g$ & Time $2 \mathrm{M} / \mathrm{SD}$ & Time $3 \mathrm{M} / \mathrm{SD}$ & $\mathrm{T} 2$ to $\mathrm{T} 3$ adjusted $b^{*}$ & Hedges' $g$ \\
\hline Mindfulness & $4.93 / 0.54$ & $4.85 / 0.42$ & -0.07 & - & $2.14 / 0.14$ & $5.00 / 0.58$ & $2.86^{* * *}$ & - \\
\hline Distress & $10.48 / 3.25$ & $9.60 / 2.56$ & -0.88 & - & $32.12 / 3.20$ & $9.15 / 1.99$ & $-22.96 * * *$ & - \\
\hline Somatization & $2.84 / 1.21$ & $2.32 / 0.95$ & -0.52 & - & $9.19 / 1.60$ & $2.42 / 0.86$ & $-6.77 * * *$ & - \\
\hline Depression & $2.92 / 1.26$ & $3.00 / 1.26$ & 0.08 & - & $10.08 / 1.52$ & $2.88 / 1.03$ & $-7.19 * * *$ & - \\
\hline \multirow[t]{2}{*}{ Anxiety } & $4.72 / 1.54$ & $4.28 / 1.24$ & -0.44 & - & $12.85 / 2.05$ & $3.85 / 1.16$ & $-9.00 * * *$ & - \\
\hline & Time $1 \mathrm{M} / \mathrm{SD}$ & Time $3 \mathrm{M} / \mathrm{SD}$ & $\mathrm{T} 1$ to $\mathrm{T} 3$ adjusted $b^{*}$ & Hedges' $g^{* *}$ & Time $1 \mathrm{M} / \mathrm{SD}$ & Time $3 \mathrm{M} / \mathrm{SD}$ & T1 to T3 adjusted $b^{*}$ & Hedges' $g^{* *}$ \\
\hline Mindfulness & $2.06 / 0.28$ & $4.85 / 0.42$ & $1.39 * * *$ & $7.41 * * *$ & $2.12 / 0.22$ & $5.00 / 0.58$ & $1.44 * * *$ & $6.36 * * *$ \\
\hline Distress & $31.28 / 4.01$ & $9.60 / 2.56$ & $-10.83 * * *$ & $6.02 * * *$ & $31.82 / 3.27$ & $9.15 / 1.99$ & $-11.34 * * *$ & $7.94 * * *$ \\
\hline Somatization & $9.38 / 1.54$ & $2.32 / 0.95$ & $-3.53 * * *$ & $5.05 * * *$ & $9.57 / 1.67$ & $2.42 / 0.86$ & $-3.58 * * *$ & $5.28 * * *$ \\
\hline Depression & $9.83 / 1.07$ & $3.00 / 1.26$ & $-3.41 * * *$ & $5.50 * * *$ & $9.86 / 1.18$ & $2.88 / 1.03$ & $-3.50 * * *$ & $6.26 * * *$ \\
\hline Anxiety & $12.07 / 2.48$ & $4.28 / 1.24$ & $-3.89 * * *$ & $4.07 * * *$ & $12.39 / 1.91$ & $3.85 / 1.16$ & $-4.27 * * *$ & $5.36 * * *$ \\
\hline
\end{tabular}

*Within-group treatment effect was evaluated controlling for age, educational background, and gender. Full information maximum likelihood estimation was used to run the model to address missing value

**Hedges' $g_{\text {corrected }}$ results calculated the effect size of treatment within-group comparison from time 1 to time 3 using only complete cases

$* * * p<0.001$

between a negative event and repeated thinking and affect (Glomb et al., 2011). Although this could likely be the reason contributing to participants' improvement in this trial study, this hypothesized change mechanism of a mindfulness-based intervention for psychological distress remains speculative due to lack of empirical data support.

Adding to existing studies about the intervention of psychological distress, which has primarily focused on individuals' affect/emotion, such as hope (Zhang et al., 2018), findings of this study support the use of mindfulness to directly intervention individuals' cognitive process and behaviors for psychological distress among stay-at-home residents during COVID-19. Furthermore, although we did not directly test for the possible mediating role of mindfulness, it is reasonable to expect that improvement in mindfulness reduces one's tendency to ruminate and increases the ability to detach from an adverse event (i.e., the ongoing COVID-19), which may result in reduced psychological distress.

\section{Limitations and Future Research Directions}

This study has some limitations. First, the study used convenience snowball sampling with adults, which led to a concern about external generalizability. Future studies should test the intervention's effectiveness using larger samples and more diverse samples across the developmental spectrum (Xiang et al., 2020). Second, we used waitlist control in our study, which may introduce additional effects to the "true effects" of the intervention condition. For example, a portion of the participants' improvement in the treatment condition may simply be due to attention or additional opportunities to socialize rather than the intervention. Given the nature of waitlist control as part of our design, we could not rule out these possibilities.

Third, the findings of this study are subject to common methods bias, e.g., the measurement context effects, which refer to the fact that measures of different constructs administered at the same time point may produce artifactual covariance independent of the content of the constructs themselves (Podsakoff et al., 2003). Similar studies in the future are encouraged to address this challenge through procedural remedies with well-designed measurement protocols if feasible. Fourth, this study measured mindfulness using the MAAS, which does not effectively measure the acceptance and nonjudgmental awareness aspects of mindfulness (Baer et al., 2006; Sauer et al., 2013). Mindfulness is a multidimensional construct, yet, MAAS only effectively measures the "acting with awareness" aspect of mindfulness, which omits a comprehensive evaluation of mindfulness in this study. Finally, the evaluation of long-term change is another limitation in 
the current study as we just monitored the practice of mindfulness during the intervention. If the participants can practice mindfulness in their daily life after the intervention, the benefits of the intervention will be further improved. Thus, future studies are encouraged to examine the long-term effect for the participants with continuous engagement in mindfulness practices.

Future studies are encouraged to evaluate online-based mindfulness interventions targeting all aspects of mindfulness and assess if the online format is more effective in improving certain aspects of mindfulness than others. Moreover, nuanced analyses evaluating certain aspects of mindfulness-based interventions concerning psychological distress will contribute to the understanding of core aspects of mindfulness that improve individuals' psychological distress.

Acknowledgements The present research was supported by Special Project of Central China Think Tank of Huazhong University of Science and Technology (The psychological adaptation and its intervention among Chinese residents staying at home during COVID-19 outbreak, Grant No. 2020HZZK014) and The Project of the National Natural Science Foundation of China (Grant No. 71901098).

Author Contributions HZ: designed and executed the study and wrote all sections the paper. AZ: conceptualized the study, assisted in data analysis, and wrote all sections of the paper. CBL: designed and executed the study and proofread the paper. JX: executed the study and proofread the study. KW: conceptualized and conducted the data analysis and wrote the methods and results of the paper.

\section{Declarations}

Ethics Approval and Consent to Participate This study was approved by the Institutional Review Board at Huazhong University of Science and Technology. All participants willingly signed an informed consent form, which was approved by the IRB at Huazhong University of Science and Technology. Due to the time-sensitive and urgent nature of the COVID19 outbreak, the study was conducted without a pre-trial registration.

Conflict of Interest The authors declare no conflict of interest.

\section{References}

Ahern, E., Kinsella, S., \& Semkovska, M. (2018). Clinical efficacy and economic evaluation of online cognitive behavioral therapy for major depressive disorder: A systematic review and meta-analysis. Expert Review of Pharmacoeconomics \& Outcomes Research, 18(1), 25-41. https://doi.org/10.1080/14737167.2018.1407245.

Baer, R. (2010). Assessing mindfulness and acceptance processes in clients: Illuminating the theory and practice of change. New Harbinger Publications.

Baer, R. A., Smith, G. T., Hopkins, J., Krietemeyer, J., \& Toney, L. (2006). Using self-report assessment methods to explore facets of mindfulness. Assessment, 13(1), 27-45. https://doi.org/10.1177/ 1073191105283504.

Bao, Y., Sun, Y., Meng, S., Shi, J., \& Lu, L. (2020). 2019-nCoV epidemic: Address mental health care to empower society. The Lancet,
395(10224), e37-e38. https://doi.org/10.1016/S0140-6736(20) 30309-3.

Brown, K. W., \& Ryan, R. M. (2003). The benefits of being present: Mindfulness and its role in psychological well-being. Journal of Personality and Social Psychology, 84(4), 822-848. https://doi. org/10.1037/0022-3514.84.4.822.

Browne, R. H. (1995). On the use of a pilot sample for sample size determination. Statistics in Medicine, 14(17), 1933-1940. https:// doi.org/10.1002/sim.4780141709.

Carpenter, J. K., Andrews, L. A., Witcraft, S. M., Powers, M. B., Smits, J. A. J., \& Hofmann, S. G. (2018). Cognitive behavioral therapy for anxiety and related disorders: A meta-analysis of randomized placebo-controlled trials. Depression and Anxiety, 35(6), 502-514. https://doi.org/10.1002/da.22728.

Chiesa, A., Anselmi, R., \& Serretti, A. (2014). Psychological mechanisms of mindfulness-based interventions: What do we know? Holistic Nursing Practice, 28(2), 124-148. https://doi.org/10.1097/ HNP.0000000000000017.

Chisholm, D., Sweeny, K., Sheehan, P., Rasmussen, B., Smit, F., Cuijpers, P., \& Saxena, S. (2016). Scaling-up treatment of depression and anxiety: A global return on investment analysis. The Lancet Psychiatry, 3(5), 415-424. https://doi.org/10.1016/S2215-0366(16) 30024-4.

Coffey, K. A., \& Hartman, M. (2008). Mechanisms of action in the inverse relationship between mindfulness and psychological distress. Complementary Health Practice Review, 13(2), 79-91. https://doi.org/10.1177/1533210108316307.

Davison, K. M., Lung, Y., Lin, S. L., Tong, H., Kobayashi, K. M., \& Fuller-Thomson, E. (2020). Psychological distress in older adults linked to immigrant status, dietary intake, and physical health conditions in the Canadian Longitudinal Study on Aging (CLSA). Journal of Affective Disorders, 265, 526-537. https://doi.org/10. 1016/j.jad.2020.01.024.

de Vibe, M., Bjørndal, A., Fattah, S., Dyrdal, G. M., Halland, E., \& Tanner-Smith, E. E. (2017). Mindfulness-based stress reduction (MBSR) for improving health, quality of life and social functioning in adults: A systematic review and meta-analysis. Campbell Systematic Reviews, 13(1), 1-264. https://doi.org/10.4073/csr. 2017.11.

Deng, Y.-Q., Li, S., Tang, Y. Y., Zhu, L. H., Ryan, R., \& Brown, K. (2012). Psychometric properties of the Chinese translation of the mindful attention awareness scale (MAAS). Mindfulness, 3(1), 10-14. https://doi.org/10.1007/s12671-011-0074-1.

Deyo, M., Wilson, K. A., Ong, J., \& Koopman, C. (2009). Mindfulness and rumination: Does mindfulness training lead to reductions in the ruminative thinking associated with depression? Explore, 5(5), 265271. https://doi.org/10.1016/j.explore.2009.06.005.

Figueroa, C. A., DeJong, H., Mocking, R. J. T., Fox, E., Rive, M. M., Schene, A. H., Stein, A., \& Ruhé, H. G. (2019). Attentional control, rumination and recurrence of depression. Journal of Affective Disorders, 256, 364-372. https://doi.org/10.1016/j.jad.2019.05. 072.

Gao, H. (2020). Anxiety and relief experience among Hubei residents under COVID-19. Chinese Social Survey. https://mp.weixin.qq. com/s?_biz=MzI3NDkyMzY4MQ==\&mid=2247485334\&idx= $1 \& \mathrm{sn}=\overline{80} 91 \mathrm{a} 7 \mathrm{~b} 6 \mathrm{f} 4 \mathrm{cc} 3 \mathrm{bcc} 16360155 \mathrm{a} 211 \mathrm{~d} 6 \mathrm{cc} \& \operatorname{source}=41 \#$ wechat_redirect

Garland, E. L., Farb, N. A., Goldin, P. R., \& Fredrickson, B. L. (2015). The mindfulness-to-meaning theory: Extensions, applications, and challenges at the attention-appraisal-emotion interface. Psychological Inquiry, 26(4), 377-387. https://doi.org/10.1080/ 1047840X.2015.1092493.

Glomb, T. M., Duffy, M. K., Bono, J. E., \& Yang, T. (2011). Mindfulness at work. In A. Joshi, H. Liao, \& J. J. Martocchio (Eds.), Research in personnel and human resources management (pp. 115-157). 
Emerald Group Publishing Limited. https://doi.org/10.1108/S07427301(2011)0000030005.

Jing, Z., Li, J., Wang, Y., Ding, L., Tang, X., Feng, Y., \& Zhou, C. (2020). The mediating effect of psychological distress on cognitive function and physical frailty among the elderly: Evidence from rural Shandong, China. Journal of Affective Disorders, 268, 88-94. https://doi.org/10.1016/j.jad.2020.03.012.

Kabat-Zinn, J. (1982). An outpatient program in behavioral medicine for chronic pain patients based on the practice of mindfulness meditation: Theoretical considerations and preliminary results. General Hospital Psychiatry, 4(1), 33-47. https://doi.org/10.1016/01638343(82)90026-3.

Kiburz, K. M., Allen, T. D., \& French, K. A. (2017). Work-family conflict and mindfulness: Investigating the effectiveness of a brief training intervention. Journal of Organizational Behavior, 38(7), 10161037. https://doi.org/10.1002/job.2181.

Langdon, S., Jones, F. W., Hutton, J., \& Holttum, S. (2011). A groundedtheory study of mindfulness practice following mindfulness-based cognitive therapy. Mindfulness, 2(4), 270-281. https://doi.org/10. 1007/s12671-011-0070-5.

Li, W., Yang, Y., Liu, Z. H., Zhao, Y. J., Zhang, Q., Zhang, L., Cheung, T., \& Xiang, Y. T. (2020). Progression of mental health services during the COVID-19 outbreak in China. International Journal of Biological Sciences, 16(10), 1732-1738. https://doi.org/10.7150/ ijbs. 45120 .

Lönnberg, G., Jonas, W., Unternaehrer, E., Bränström, R., Nissen, E., \& Niemi, M. (2020). Effects of a mindfulness based childbirth and parenting program on pregnant women's perceived stress and risk of perinatal depression - Results from a randomized controlled trial. Journal of Affective Disorders, 262, 133-142. https://doi.org/10. 1016/j.jad.2019.10.048.

Ma, Y., She, Z., Siu, A. F. Y., Zeng, X., \& Liu, X. (2018). Effectiveness of online mindfulness-based interventions on psychological distress and the mediating role of emotion regulation. Frontiers in Psychology, 9, 2090. https://doi.org/10.3389/fpsyg.2018.02090.

Mazzer, K., Boersma, K., \& Linton, S. J. (2019). A longitudinal view of rumination, poor sleep and psychological distress in adolescents. Journal of Affective Disorders, 245, 686-696. https://doi.org/10. 1016/j.jad.2018.11.053.

Nakamura, K., Watanabe, Y., Kitamura, K., Kabasawa, K., \& Someya, T. (2019). Psychological distress as a risk factor for dementia after the 2004 Niigata - Chuetsu earthquake in Japan. Journal of Affective Disorders, 259, 121-127. https://doi.org/10.1016/j.jad.2019.08. 041

Nishi, D., Susukida, R., Usuda, K., Mojtabai, R., \& Yamanouchi, Y. (2018). Trends in the prevalence of psychological distress and the use of mental health services from 2007 to 2016 in Japan. Journal of Affective Disorders, 239, 208-213. https://doi.org/10.1016/j.jad. 2018.07.016

Perez-Blasco, J., Viguer, P., \& Rodrigo, M. F. (2013). Effects of a mindfulness-based intervention on psychological distress, well-being, and maternal self-efficacy in breast-feeding mothers: Results of a pilot study. Archives of Women's Mental Health, 16(3), 227-236. https://doi.org/10.1007/s00737-013-0337-z.

Podsakoff, P. M., MacKenzie, S. B., Lee, J. Y., \& Podsakoff, N. P. (2003). Common method biases in behavioral research: A critical review of the literature and recommended remedies. Journal of Applied Psychology, 88(5), 879-903. https://doi.org/10.1037/ 0021-9010.88.5.879.

Recklitis, C. J., Blackmon, J. E., \& Chang, G. (2017). Validity of the Brief Symptom Inventory-18 (BSI-18) for identifying depression and anxiety in young adult cancer survivors: Comparison with a Structured Clinical Diagnostic Interview. Psychological Assessment, 29(10), 1189-1200. https://doi.org/10.1037/ pas0000427.
Ridner, S. H. (2004). Psychological distress: Concept analysis. Journal of Advanced Nursing, 45(5), 536-545. https://doi.org/10.1046/j.13652648.2003.02938.x.

Sauer, S., Walach, H., Schmidt, S., Hinterberger, T., Lynch, S., Büssing, A., \& Kohls, N. (2013). Assessment of mindfulness: Review on state of the art. Mindfulness, 4(1), 3-17. https://doi.org/10.1007/ s12671-012-0122-5.

Shapiro, S. L., Carlson, L. E., Astin, J. A., \& Freedman, B. (2006). Mechanisms of mindfulness. Journal of Clinical Psychology, 62(3), 373-386. https://doi.org/10.1002/jclp.20237.

Shapiro, S. L., Schwartz, G. E., \& Bonner, G. (1998). Effects of mindfulness-based stress reduction on medical and premedical students. Journal of Behavioral Medicine, 21(6), 581-599. https://doi. org/10.1023/A:1018700829825.

Sharma, M., \& Rush, S. E. (2014). Mindfulness-based stress reduction as a stress management intervention for healthy individuals: A systematic review. Journal of Evidence-Based Complementary \& Alternative Medicine, 19(4), 271-286. https://doi.org/10.1177/ 2156587214543143

Shigemura, J., Ursano, R. J., Morganstein, J. C., Kurosawa, M., \& Benedek, D. M. (2020). Public responses to the novel 2019 coronavirus (2019-nCoV) in Japan: Mental health consequences and target populations. Psychiatry and Clinical Neurosciences, 74(4), 281282. https://doi.org/10.1111/pcn.12988.

Spijkerman, M. P. J., Pots, W. T. M., \& Bohlmeijer, E. T. (2016). Effectiveness of online mindfulness-based interventions in improving mental health: A review and meta-analysis of randomised controlled trials. Clinical Psychology Review, 45, 102-114. https://doi. org/10.1016/j.cpr.2016.03.009.

Tanji, F., Sugawara, Y., Tomata, Y., Watanabe, T., Sugiyama, K., Kaiho, Y., Tomita, H., \& Tsuji, I. (2017). Psychological distress and the incident risk of functional disability in elderly survivors after the Great East Japan earthquake. Journal of Affective Disorders, 221, 145-150. https://doi.org/10.1016/j.jad.2017.06.030.

Wang, C., Pan, R., Wan, X., Tan, Y., Xu, L., Ho, C. S., \& Ho, R. C. (2020a). Immediate psychological responses and associated factors during the initial stage of the 2019 coronavirus disease (COVID-19) epidemic among the general population in China. International Journal of Environmental Research and Public Health, 17(5), 1729. https://doi.org/10.3390/ijerph17051729.

Wang, J., Kelly, B. C., Liu, T., Zhang, G., \& Hao, W. (2013). Factorial structure of the brief symptom inventory (BSI)-18 among Chinese drug users. Drug and Alcohol Dependence, 133(2), 368-375. https://doi.org/10.1016/j.drugalcdep.2013.06.017.

Wang, K., \& Bishop, N. J. (2019). Social support and monetary resources as protective factors against food insecurity among older Americans: Findings from a health and retirement study. Food Security, 11(4), 929-939. https://doi.org/10.1007/s12571-019-00945-8.

Wang, K., Chu, Y., \& Lombe, M. (2020b). Utilization of informal food support among low-income households: Application of Andersen's service use model. Families in Society, 101(3), 368-381. https://doi. org/10.1177/1044389419860636.

Watanabe, N., Horikoshi, M., Shinmei, I., Oe, Y., Narisawa, T., Kumachi, M., Matsuoka, Y., Hamazaki, K., \& Furukawa, T. A. (2019). Brief mindfulness-based stress management program for a better mental state in working populations-Happy nurse project: A randomized controlled trial. Journal of Affective Disorders, 251, 186-194. https://doi.org/10.1016/j.jad.2019.03.067.

Xiang, Y. T., Yang, Y., Li, W., Zhang, L., Zhang, Q., Cheung, T., \& Ng, C. H. (2020). Timely mental health care for the 2019 novel coronavirus outbreak is urgently needed. The Lancet Psychiatry, 7(3), 228 229. https://doi.org/10.1016/S2215-0366(20)30046-8.

Zhang, A., Borhneimer, L. A., Weaver, A., Franklin, C., Hai, A. H., Guz, S., \& Shen, L. (2019). Cognitive behavioral therapy for primary care depression and anxiety: A secondary meta-analytic review using robust variance estimation in meta-regression. Journal of 
Behavioral Medicine, 42(6), 1117-1141. https://doi.org/10.1007/ s10865-019-00046-z.

Zhang, A., Ji, Q., Currin-McCulloch, J., Solomon, P., Chen, Y., Li, Y., Jones, B., Franklin, C., \& Nowicki, J. (2018). The effectiveness of solution-focused brief therapy for psychological distress among Chinese parents of children with a cancer diagnosis: A pilot randomized controlled trial. Supportive Care in Cancer, 26(8), 2901-2910. https://doi.org/10.1007/s00520-018-4141-1.

Zhang, A., Hu, R., Wang, K., \& Antalis, E. P. (2020a). Age moderates the association between psychological distress and engagement in mindfulness among cancer patients and survivors: A population- based study. Journal of Psychosocial Oncology, 38(5), 513-526. https://doi.org/10.1080/07347332.2020.1764158.

Zhang, Y., Xue, J., \& Huang, Y. (2020b). A meta-analysis: Internet mindfulness-based interventions for stress management in the general population, e20493. Medicine, 99(28). https://doi.org/10.1097/ MD.0000000000020493.

Publisher's Note Springer Nature remains neutral with regard to jurisdictional claims in published maps and institutional affiliations. 